Review

\title{
Phase-Change Nanoparticles Using Highly Volatile Per- fluorocarbons: Toward a Platform for Extravascular Ultrasound Imaging
}

\author{
Terry O. Matsunaga $1^{* \otimes}$ and Paul S. Sheeran ${ }^{2 *}$, Samantha Luois ${ }^{3}$, Jason E. Streeter ${ }^{2}$, Lee B. Mullin², Bhaskar \\ Banerjee $^{4}$, and Paul A. Dayton ${ }^{2}$ \\ 1. Department of Medical Imaging, University of Arizona, Tucson, AZ 85724, USA; \\ 2. Joint Department of Biomedical Engineering, The University of North Carolina and North Carolina State University, Chapel Hill, NC \\ 27599, USA; \\ 3. Undergraduate Biology Research Program, University of Arizona, Tucson, AZ 85724, USA; \\ 4. Department of Gastroenterology, Optical Sciences, and Biomedical Engineering, University of Arizona, Tucson, AZ 85724, USA. \\ * Authors contributed equally to this work.
}

\begin{abstract}
$\bowtie$ Corresponding author: Email: tmatsunaga@radiology.arizona.edu
(c) Ivyspring International Publisher. This is an open-access article distributed under the terms of the Creative Commons License (http://creativecommons.org/ licenses/by-nc-nd/3.0/). Reproduction is permitted for personal, noncommercial use, provided that the article is in whole, unmodified, and properly cited.
\end{abstract}

Received: 2012.07.09; Accepted: 2012.1 I.02; Published: 2012.12.23

\begin{abstract}
Recent efforts using perfluorocarbon (PFC) nanoparticles in conjunction with acoustic droplet vaporization has introduced the possibility of expanding the diagnostic and therapeutic capability of ultrasound contrast agents to beyond the vascular space. Our laboratories have developed phase-change nanoparticles (PCNs) from the highly volatile PFCs decafluorobutane (DFB, bp $=-2{ }^{\circ} \mathrm{C}$ ) and octafluoropropane (OFP, bp $=-37^{\circ} \mathrm{C}$ ) for acoustic droplet vaporization. Studies with commonly used clinical ultrasound scanners have demonstrated the ability to vaporize PCN emulsions with frequencies and mechanical indices that may significantly decrease tissue bioeffects. In addition, these contrast agents can be formulated to be stable at physiological temperatures and the perfluorocarbons can be mixed to modulate the balance between sensitivity to ultrasound and general stability. We herein discuss our recent efforts to develop finely-tuned diagnostic/molecular imaging agents for tissue interrogation. We discuss studies currently under investigation as well as potential diagnostic and therapeutic paradigms that may emerge as a result of formulating PCNs with low boiling point PFCs.
\end{abstract}

Key words: Phase-change nanoparticles, extravascular imaging, ultrasound, perfluorocarbon, Acoustic droplet vaporization, diagnostic, therapeutic.

\section{Introduction}

Since their first use in ultrasonography by Gramiak and Shah over 40 years ago, the utility of microbubble-based contrast agents (MCA) in the clinical and research arenas has spanned both diagnostic and therapeutic applications [1-8]. Today ultrasound remains a highly desirable methodology for diagnostic imaging primarily due to its portability, and offers advantages over other imaging modalities because of its real-time, non-ionizing, high frame-rate imaging. Furthermore, ultrasound remains a low-cost modality that continues to decrease in system price with advances in manufacturing technology [9]. While advantageous in many respects, ultrasound currently lags behind other modalities with regard to 
the ability to use contrast agents for interrogation of the interstitial space and the physiologic processes occurring there. In PET/SPECT/MRI, diagnosing complex extravascular diseases can be approached with radionuclides or paramagnetic contrast agents [10].

In ultrasound imaging, there is great interest in designing contrast agents that can investigate sites of inflammation and solid tumors that result in highly permeable vascular networks [11-13]. However, ultrasound contrast agents currently approved for clinical applications are produced in sizes that inherently restrict the particles to flow only through the vascular networks. Formulations of MCAs are typically designed to balance a number of trade-offs involved with in vivo contrast imaging. First, because MCAs are typically administered through intravenous injection, they must be able to freely traverse capillary beds, and therefore must be similar to, or smaller than, the size of red blood cells (approximately $8 \mu \mathrm{m}$ in diameter) [14]. Second, reflected energy that results in imaging contrast is a function of both the scattering cross section (larger diameters resulting in greater contrast) and the microbubble's natural resonance $[15,16]$. Most studies have found that microbubbles in the several-micron diameter range produce a strong contrast signal at clinically relevant imaging frequencies, however, this size distribution also precludes MCAs from diffusing through 'leaky' tumor vasculature [17].

Researchers have attempted to overcome these limitations by designing nanoparticles still capable of some degree of echogenicity, such as gas-filled nanobubbles and perfluorocarbon (PFC) nanodroplets on the order of 100-500 nm - conceivably small enough to extravasate into permeable tumor types [18-24]. Nanoemulsions have been used in the past as drug delivery agents in conjunction with ultrasound-mediated localized delivery via radiation force/acoustic streaming [25]. The fact that these particles are often encapsulated in lipid shells may serve additional benefits - as outlined by Hendrickson and Lyon - in that soft colloids may have the ability to "squeeze" through pores due to additional elasticity [26]. Thus, it is possible that soft-shelled, lipid-coated, liquid-core perfluorocarbon nanoparticles may be able to extravasate through pores of significantly smaller size than their diameter would dictate. However, sub-micron bubbles produce substantially less image contrast than those in the micron size range for the same concentration and acoustic parameters.

PFC droplets made from low boiling point compounds represent a unique approach in that they may be acoustically triggered to vaporize (typically termed acoustic droplet vaporization or ADV) to form bubbles much larger than the original droplet size combining small initial particle size with high echogenicity. ADV has been proposed for a number of applications at both the microscale and nanoscale, such as selective vessel occlusion and various other forms of therapy and drug/gene delivery [27-29].

Much of the intellectual groundwork for nanoscale ADV particles was formed in two early patents and more recent publications from several researchers [30-35]. Expanding on ideas posited in the patent literature, researchers proposed the concept of a nanoemulsion small enough to accumulate at an interstitial target site and capable of being converted to the gaseous state through the application of ultrasonic energy and used to aid in therapeutic dose delivery. While in the liquid phase, these perfluorocarbon nanoparticles can be generated in the low 100s of nanometers, and can expand once activated to form gas-filled microbubbles on the order of 3-10 times larger in diameter depending upon whether they are in an air-saturated or degassed environment [31, 36, 37].

Most previous studies of nanoscale ADV particles have relied upon the use of PFC compounds with boiling points above room temperature so as to maintain stability [31]. The lowest boiling perfluorocarbon core used until recently has been dodecafluoropentane (DDFP, bp $=29^{\circ} \mathrm{C}$ ) while other larger perfluorinated molecules such as perfluorohexane $\left(\mathrm{bp}=57^{\circ} \mathrm{C}\right.$ ) have been used as well. For many applications, these compounds appear to be sufficiently susceptible to ultrasound to be activated at feasible acoustic parameters. However, as particle size moves towards the low 100s of nanometers, the amount of acoustic energy needed increases significantly as a result of additional Laplace pressure on the droplet core - effectively increasing the boiling point and acoustic energy needed to cause phase transition [32, 36]. This increase in activation energy makes approaching diagnostic and molecular imaging applications with nanoscale ADV agents especially difficult. Recent reports have suggested that this effect is so great for some formulations that recently vaporized bubbles may even re-condense to the liquid state in the absence of ultrasound or cannot be vaporized with the full acoustic power of the ultrasound transducer $[38,39]$.

One possibility for increasing the sensitivity of these types of agents is to develop them from compounds more volatile than previously considered. By the same effect that allows DDFP to form nano- and microscale droplets that remain in the liquid state at temperatures much higher than their normal boiling point, droplets might be formed from perfluorocar- 
bons such as DFB and OFP [40]. Figure 1 shows the effect of perfluorocarbon boiling point as a function of pressure $[32,36]$. Note that the boiling points of perfluorohexane and DDFP exceed their standard boiling points found under standard temperature and pressure quite substantially. While the curves suggest that microscale droplet of DFB and OFP would likely be highly unstable, droplets formed from these highly volatile fluorocarbons at the nanoscale may be stabilized and vaporized with significantly less energy than their counterparts formulated from less volatile perfluorocarbons.

Using this as a rationale for development, we set out to demonstrate stabilization of highly volatile perfluorocarbons as a new class of dynamic, activatable biomaterials. With the assumption that lower boiling perfluorocarbons are necessary to maintain reasonable ultrasound output requirements for vaporization of $100-200 \mathrm{~nm}$ nanoparticles, we hypothesized that low boiling DFB (and later octafluoropropane) nanoparticles could be formulated and maintain their stability at physiologic temperatures and that they would be subject to vaporization at lower ultrasound outputs. A pictorial diagram of the design is shown in Figure 2.

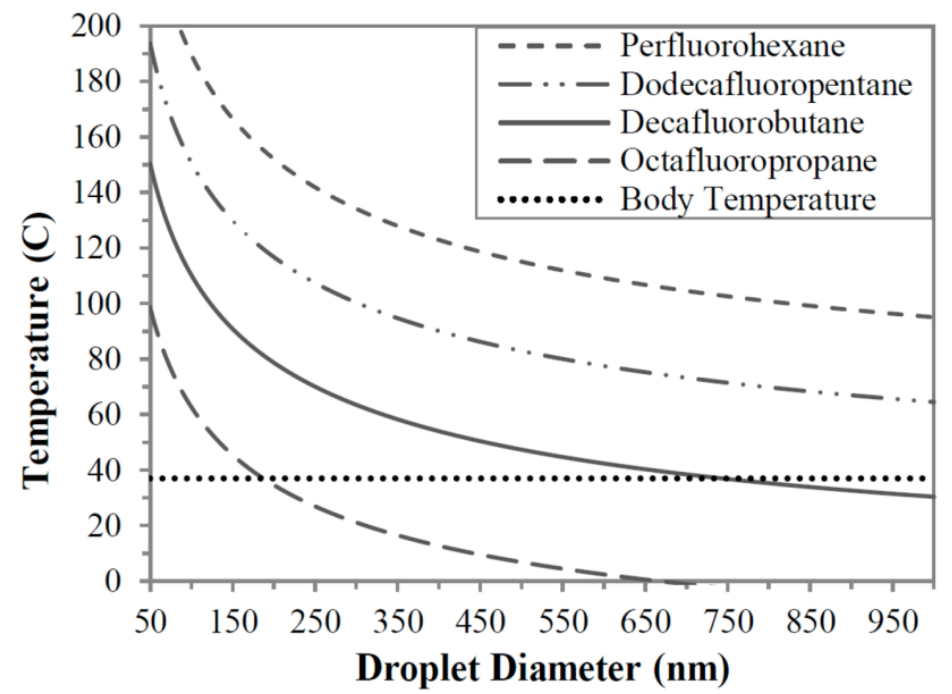

Figure I. Vaporization temperature predictions for perfluorocarbon particles as a function of droplet diameters. Reprinted with permission from Langmuir. Copyright 201 I American Chemical Society.

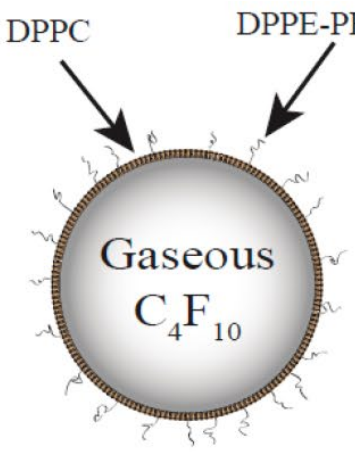

Microbubble Preparation

(Agitation, Sonication)

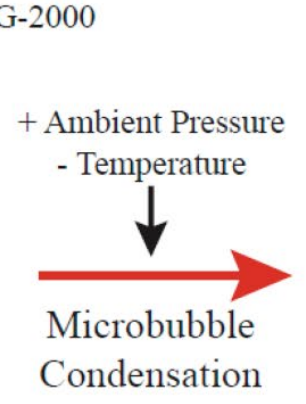

$$
\text { Liquid } \mathrm{C}_{4} \mathrm{~F}_{10}
$$

Figure 2. Pictorial diagram for making phase-change nanoparticles of submicron size distribution using pressurization and slow cooling to condense the gas inside the microbubbles. Reprinted with permission from Langmuir. Copyright 201 I American Chemical Society. 


\section{Metastable Decafluorobutane nanoparti- cles}

Initially, the approach to form stable DFB nanoparticles was to condense the PFC compound at reduced temperatures and subsequently mix the resulting liquid aliquot with a lipid cocktail consisting of dipalmitoylphosphatidylcholine (DPPC), 1- palmitoyl-2-hydroxy-sn-glycero-3-phosphocholine (LPC) and 1,2-dipalmitoyl-sn-glycero-3 phosphoethanolamine-N-[methoxy(polyethyleneglycol)-2000] (DPPEPEG-2000). The samples could then be extruded in a cold room $\left(-20^{\circ} \mathrm{C}\right)$ using a hand-held extruder and passing through porous membranes. Our initial results demonstrated that indeed liquid DFB could be stabilized into lipid-coated particles that remained stable at body temperature, and that vaporization could occur at acoustic parameters less likely to induce cavitation-based bioeffects than alternative compounds. Figure 3 demonstrates the vaporization pressure of the largest, optically resolvable DFB droplets present in each sample as a function of particle size when exposed to $5 \mathrm{MHz}$ ultrasound in a degassed water bath. Using these large particles allowed for simple measurement of individual droplet vaporization thresholds, and made comparisons between compounds facile. As was expected, the mechanical index (MI: defined as the peak negative pressure in $\mathrm{MPa}$ divided by the square root of the center frequency in $\mathrm{MHz}$ ) decreased significantly for droplets composed of DFB compared to dodecafluoropentane and perfluoro(2-methyl-3- pentanone) [bp $49.2{ }^{\circ} \mathrm{C}$ ] - highlighting the expected relationship between reduced initial boiling point and a lower acoustic vaporization threshold.

However, despite their apparent stability and the fact that sensitivity to ultrasound was higher than other perfluorocarbons, the size of the DFB particles generated by extrusion were primarily in the micron range regardless of membrane pore size, and the extrusion method appeared somewhat unreliable with respect to making predominantly smaller particles. This may be due to secondary effects such as aggregation, coalescence, or Ostwald Ripening. Alternatively, the malleability of the liquid DFB along with the very low surface tension could deform through the filter pores, forming a droplet much larger than the filter pore diameter.

Through these initial studies, the need for uniform-sized nanoparticles that were also resistant to aggregation, coalescence and/or Ostwald ripening became apparent [41]. One possible method that was entertained was based upon microbubble sizing studies by Borden and coworkers who found that upon administration of pressure at approximately 100 $\mathrm{kPa}$ of ambient pressure for nine cycles, the microbubble size would "jump" to a smaller size distribution [42]. Based upon this knowledge, we hypothesized that if pressure alone would promote a smaller size distribution, then perhaps a combination of increased pressure and reduced ambient temperature could cause DFB microbubbles to collapse and/or condense to the liquid state and form much smaller droplets.

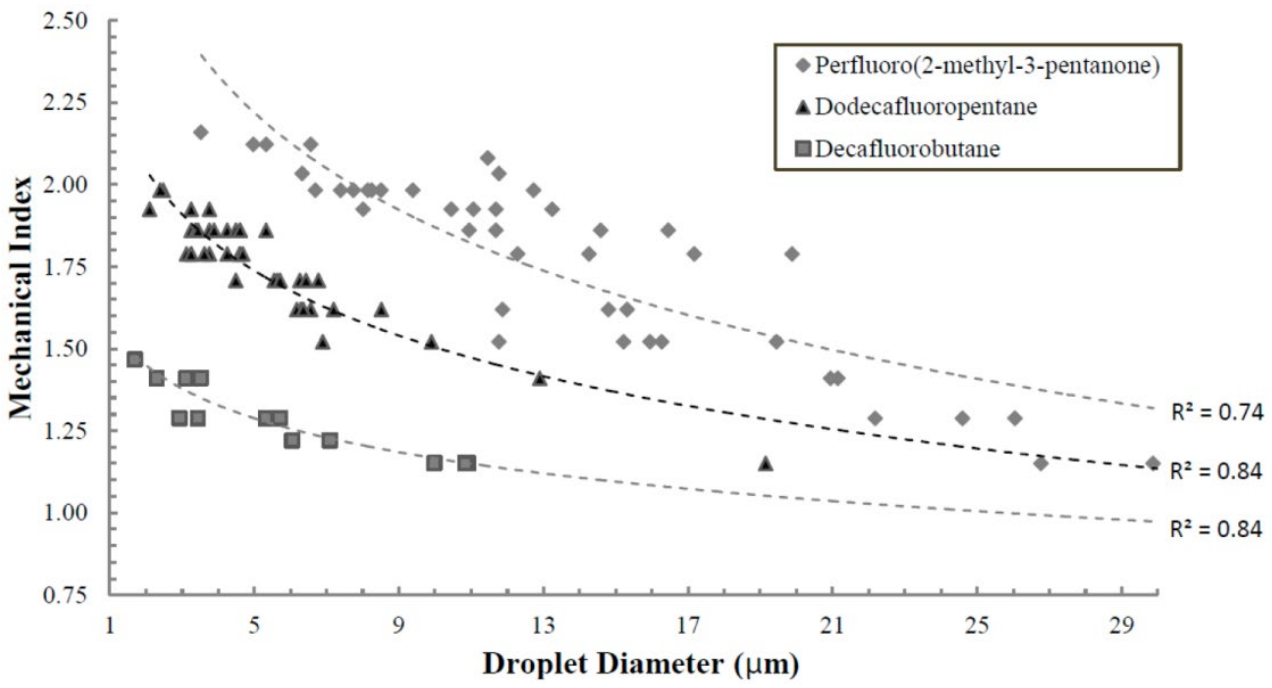

Figure 3. Ultrasound vaporization thresholds (mechanical index) of individual particles as a function of initial particle diameter when exposed to $5 \mathrm{MHz}$ ultrasound ( 10 cycles). Decafluorobutane droplets require significantly less energy than droplets composed of higher boiling point compounds. Reprinted from Ultrasound in Medicine \& Biology. Vol. 37 (9). Sheeran PS, Wong VP, Luois S et al. De- 
cafluorobutane as a Phase-Change Contrast Agent for Low-Energy Extravascular Ultrasonic Imaging. Pgs. I5I8-I530, Copyright 20II, with permission from Elsevier. *note: rarefactional pressure (in MPa) can be calculated from the mechanical index with $P_{n e g}=M I \times \sqrt{5}$

This method of 'microbubble condensation' utilizing ambient pressures on the order of $300-500 \mathrm{kPa}$ and cooling below $0{ }^{\circ} \mathrm{C}$, successfully generated DFB PCNs with a smaller distribution as determined by particle sizing methodology. As can be seen in Figure 4 , the size distribution after applying pressure and reduced temperature at three lipid concentrations yielded a particle distribution on the order of $200 \mathrm{~nm}$ to $300 \mathrm{~nm}$ in diameter. More recent studies have shown that similar results can be achieved with much lower ambient pressures - using cooling as the primary driving force of microbubble condensation [43].

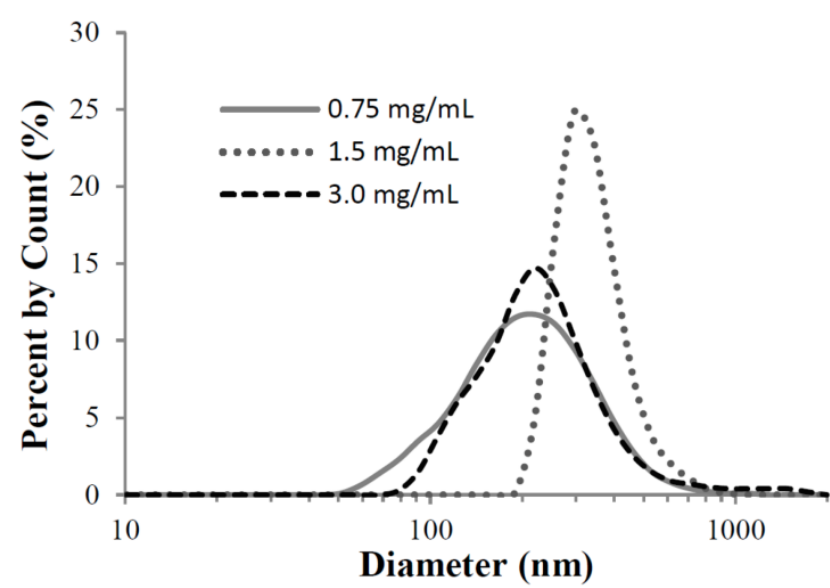

Figure 4. Submicron particle sizing of nanodroplets produced by the microbubble condensation method. Peak sizes of $200-300 \mathrm{~nm}$ resulted, which appeared to be independent of lipid concentration. Mean droplet size for each curve in order of increasing lipid concentration was $229 \pm 120,345 \pm 97$, and $325 \pm 268 \mathrm{~nm}$, respectively. Mode droplet size was 220, 295, and $255 \mathrm{~nm}$, respectively. Reprinted with permission from Langmuir. Copyright 20II American Chemical Society.

Subsequent vaporization of the PCNs by acoustic energy yielded a majority of microbubbles in the 1 - 2 micron range (Figure 5). These results show the distribution of vaporized microbubbles as a function of two different mechanical indices $(\mathrm{MI}=1.2$ and 1.7, or $2.7 \mathrm{MPa}$ and $3.8 \mathrm{MPa}$ ) at a $5 \mathrm{MHz}$ center frequency. As the MI is increased, the resultant distribution skews towards smaller diameters, likely due to increased efficiency in vaporization of the small droplets. It is also noted that this distribution was achieved by increasing the total lipid concentration to $3 \mathrm{mg} / \mathrm{mL}$ of total lipid in the formulation, possibly an indication of more stabilized PCNs in the milieu, though most of our ongoing studies use lipid concentrations near 1 $\mathrm{mg} / \mathrm{mL}$. In addition, the microbubble sizing profiles were very similar to the original microbubble precursor size distributions, which suggests not only that the precursors were efficiently converted to nanodroplets and could be converted back to the gaseous state, but that this technique allows the possibility of highly tailoring the resulting 'post-vaporization' microbubble distributions beyond what other generation techniques allow.

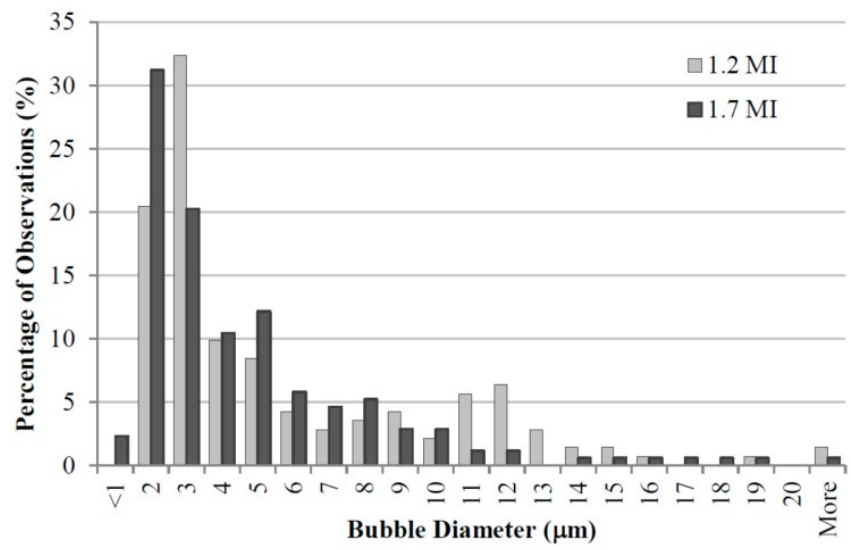

Figure 5. Microbubble diameter post vaporization as a function of acoustic pressure at $5 \mathrm{MHz}$ frequency and lipid concentration of $3 \mathrm{mg} / \mathrm{mL}$. As pressure increases, the distribution skews towards smaller resultant microbubble diameters. Reprinted with permission from Langmuir. Copyright 2011 American Chemical Society. *note: rarefactional pressure (in $\mathrm{MPa}$ ) can be calculated from the mechanical index with $P_{n e g}=M I \times \sqrt{5}$.

To our knowledge, these studies represent the first proofs-of-principle of the potential for formulating PCNs from highly volatile perfluorocarbons for ultrasound-mediated vaporization in vivo. To date, we have observed that, while some degree of spontaneous vaporization is present (thermal instability), a substantial number of droplets remain stable while incubated in cell culture for periods longer than 4 hours under physiologic conditions and can be vaporized with clinical diagnostic acoustic parameters afterward (unpublished data). More recently, this methodology was expanded to the use of an even more volatile perfluorocarbon - octafluoropropane $\left(b p=-36.7^{\circ} \mathrm{C}\right)$ at the expense of thermal stability. As was expected, the ultrasound vaporization threshold for the OFP nanoparticles was significantly lower than DFB (see Figure 6) - further confirming that a more volatile perfluorocarbon yielded a more easily 
vaporizable particle albeit at decreased stability at physiologic temperatures, and confirming the significance of the extremely high Laplace pressures at the nanoscale.

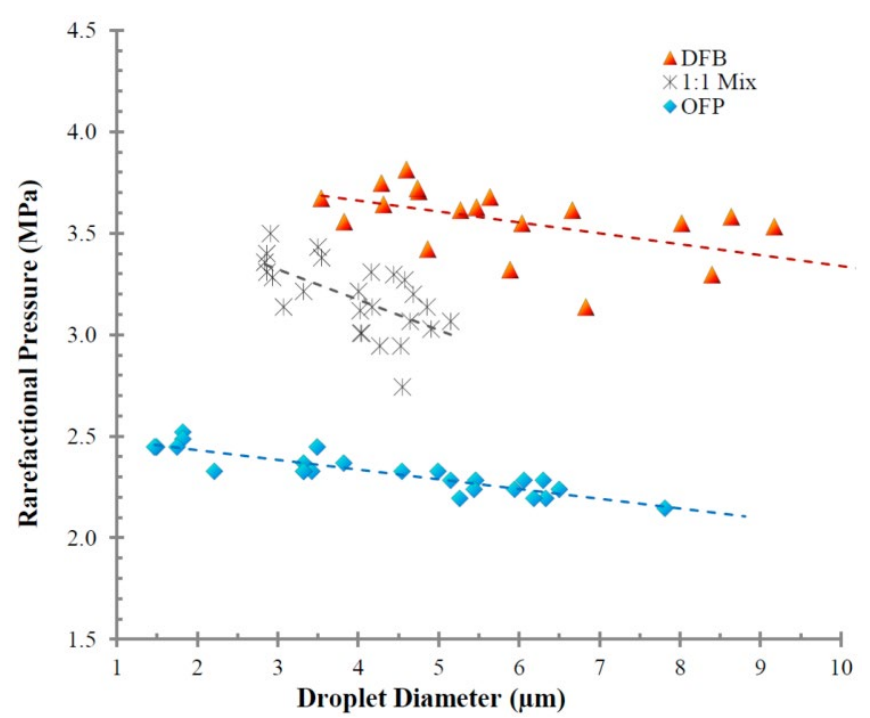

Figure 6. Rarefactional pressure required to vaporize droplets of based on initial diameter, and as a function of PFC composition (decafluorobutane, octafluoropropane and a 50:50 mixture of the two gasses). Measurements taken at room temperature $\left(22^{\circ} \mathrm{C}\right)$ using an $8 \mathrm{MHz}$ ultrasound transducer with a 2-cycle pulse length. Note the intermediate pressure required for vaporization of the mixture. Reprinted from Biomaterials. Vol. 33. Sheeran PS, Luois S, Mullin LB et al. Design of ultrasonically-activatable nanoparticles using low boiling point perfluorocarbons. pgs. 3262-68, Copyright 20II, with permission from Elsevier.

In addition, it is worth noting that, although the technique of particle generation via decreased temperature and increased ambient pressure is advantageous over extrusion-based techniques for use with lower-boiling perfluorocarbons, the methodology requires refinement. For example, we observe that upon condensation, the resulting emulsions can often contain droplets larger than 2-3 $\mu \mathrm{m}$ in diameter. These represent an insignificant part of the overall distribution, but are likely to have high significance in vivo. Large outliers in ADV emulsions vaporize much more easily than the smaller particles present, provide much higher contrast (thereby skewing data), and perhaps more importantly - can cause unwanted embolization or damage if too large. In these emulsions, the particles may result from; 1 ) a small number of very large bubbles present in the precursor bubbles; 2) larger gas pockets sequestered within the microbubble matrix; or; 3) could possibly be a result of pressure or temperature inducing Ostwald ripening or aggregation and coalescence of the microbubbles or resultant nanoparticles. However, this might be rectified by changing the rate of pressurization so as to retard any coalescence effects. In addition, elimination of large bubbles may be rectified by utilizing flow-filtering techniques upon initial microbubble distributions to achieve a more uniform size prior to condensation [16, 44]. Current efforts are focused upon understanding this phenomenon and refining the resulting distributions. Nonetheless, this method of utilizing pre-formed microbubbles is advantageous over membrane extrusion where it may not be feasible to extrude at temperatures below the boiling point of the perfluorocarbon (i.e. octafluoropropane where extrusion would have to occur at or below $-37^{\circ} \mathrm{C}$ ) well below the freezing point of the lipid colloidal solution.

An additional caveat to this discovery was the fact that a variety of different lipid combinations were used as stabilizing shells for the PCNs. In fact, virtually all combinations of lipids used to date have been successfully utilized as stabilizing shells. The implications of this flexibility in shell composition is notable, as we have used as much as 30 mole $\%$ cationic lipids (i.e. 1,2-dipalmitoyl-3-trimethylammonium propane (chloride salt; 16:0 TAP)) - which is useful for electrostatic complexing of plasmids to the nanoparticle surface, thereby making them suitable for gene delivery. In addition, the lipid shell can be complemented with other components so as to make the shells more rigid (or malleable as the case may be) thereby altering the stability and vaporizability of the PCNs.

\section{Post-Vaporization Bubble Growth Sec- ondary to Gas Diffusion}

As was described previously in ADV experiments with DDFP microdroplets by Kripfgans et al., microbubbles formed by vaporization continue to expand with time beyond ideal gas law predictions due to the influx of dissolved gas in the milieu, and has been confirmed by others with nanoparticle formulations [37, 45]. This was also observed in our studies of ADV-induced DFB microbubbles. Using both degassed and undegassed aqueous milieu, it was demonstrated that, upon acoustic vaporization, the resultant diameter of the newly formed microbubbles in the undegassed solution was significantly larger than those in the degassed solution. In fact, in the degassed milieu, the resultant diameter closely approximated that of those predicted using simple ideal gas law estimations for liquid-to-gas conversions (approx. $5-6$ fold increase in diameter). This can be seen in Figure 7. Note, that this has also been demonstrated previously by Lundgren et al. for dode- 
cafluoropentane microbubbles that were intended for use as a blood substitute $[46,47]$ as well as a nitrogen "scrubber" for potentially treating underwater nitrogen toxicity [48]. More recently, Reznik et al. (2012) demonstrated that depending on shell composition, vaporized PFC droplets may expand to between 10-50x their initial diameter over the course of 15 minutes in vitro [49].

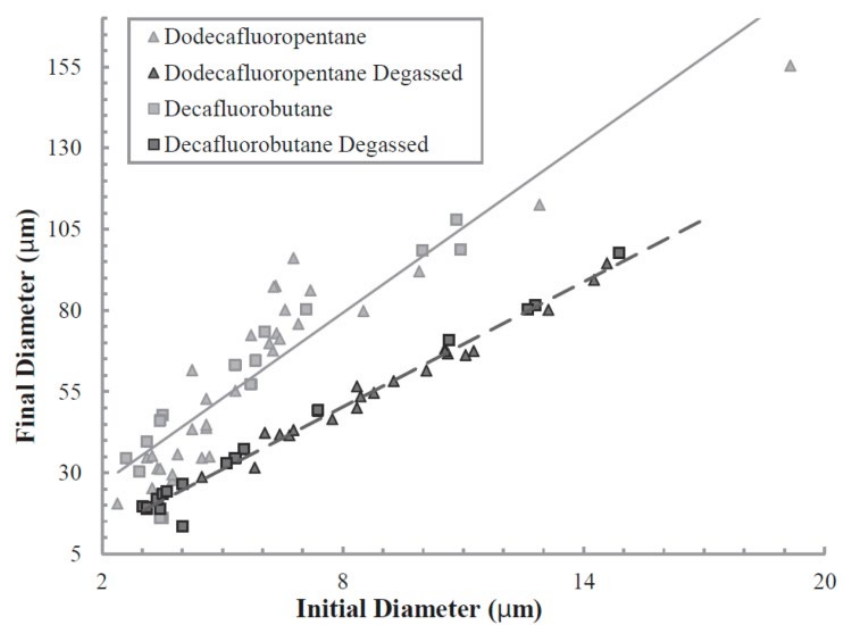

Figure 7. Corresponding Microbubble diameters (y-axis) after ultrasound-mediated vaporization of PCNs. Reprinted from UItrasound in Medicine \& Biology. Vol. 37 (9). Sheeran PS, Wong VP, Luois $S$ et al. Decafluorobutane as a Phase-Change Contrast Agent for Low-Energy Extravascular Ultrasonic Imaging. Pgs. I518-1530, Copyright 20II, with permission from Elsevier.

This post-vaporization expansion is likely to be an important component in vivo, and requires further study. In some aspects, this may be beneficial - as a larger bubble will result in increased ultrasound backscatter and higher contrast in imaging (or, alternatively, allows for use of smaller droplets) - although it may result in non-ideal bubble sizes for applications involving acoustic radiation force manipulation of the resulting bubbles (dependent on ideal resonance diameters) [50].

\section{Modulation of Ultrasound Vaporization Energy by Modifying Perfluorocarbon ratios}

Previous work by Kawabata and coworkers explored the possibility of modulating ultrasound output requirements for initiating droplet vaporization [34]. They approached this goal by the mixing of two liquid perfluorocarbons to determine if the combination would modulate the boiling point similar to an azeotrope. This same concept can be extended to modulate the stability and ultrasound sensitivity of droplets formed from highly volatile gaseous perfluorocarbons. Thus, our approach mirrored that of Kawabata et al. with the exception that it used mixtures of DFB and OFP while in the gaseous state prior to condensation. A 1:1 (v:v) mixture of DFB and OFP was purged into a vial of lipids containing 1,2-distearoyl-sn-glycero-3-phosphocholine (DSPC) and

1,2-distearoyl-sn-glycero-3-phosphoethanolamine-Nmethoxy(polyethyleneglycol)- 2000 (DSPE-PEG2000) in a 9:1 $\mathrm{M}$ ratio and a total lipid concentration of 1.0 $\mathrm{mg} / \mathrm{mL}$ [51]. Results indicated that the mixture of two volatile perfluorocarbon gases condensed together would result in a mixed liquid droplet with stability and sensitivity to ultrasound between droplets composed of each of the 'parent' compounds. Figure 6 also demonstrates the change in acoustic output as a function of either pure condensed perfluorocarbons or a 1:1 mixture. As can be seen, the 1:1 mixture modulated the vaporization rarefractional pressure to an intermediate level between the two pure gas PCNs containing OFP and DFB. This exciting result demonstrates that the vaporization parameters can be altered as a function of the gas mixture and potentially finely-tuned for specific applications.

Indeed, as was also believed, the stability of the nanoparticle could be modulated as well. Using an Accusizer 780 optical particle sizing system (Particle Sizing Systems, Santa Barbara, CA), we were able to size the nanoparticles as a function of time at two temperatures, $22{ }^{\circ} \mathrm{C}$ and $37^{\circ} \mathrm{C}$. The sizing of the nanoparticles was constrained by the limits of measurement methodology ( $\geq 500 \mathrm{~nm}$ ). However, as an estimation of stability between different formulations, this approach was justified since smaller nanoparticles subject to higher Laplace pressures will exhibit generally greater stability than the portion of the samples greater than $500 \mathrm{~nm}$. Results indicated that over the period of 60 minutes, DFB nanoparticles exhibited high stability at both temperatures with only small changes in distribution or particle count. Mixtures of 1:1 DFB and OFP exhibited a $17 \%$ decrease in mean concentration at $22{ }^{\circ} \mathrm{C}$ and $45 \%$ at $37^{\circ} \mathrm{C}$. Nanoparticles made entirely of OFP exhibited the least stability with an $82 \%$ drop in concentration over 60 minutes at both $22^{\circ} \mathrm{C}$ and $37^{\circ} \mathrm{C}$ (Figure 8). Studies are ongoing to determine whether this balance of stability and ultrasound sensitivity can be altered more favorably by mixing highly volatile PFCs (OFP, DFB) with PFCs that are in the liquid state at room temperature (DDFP, PFH). It may be that mixing a highly volatile PFC such as OFP with a higher molecular weight compound will maximize stability while retaining much of the desired sensitivity to ultrasound. 
Interestingly, the above results demonstrate an important phenomenon worth discussion at this time; that being that combination of perfluorocarbon mixtures based upon their volatility allows one to modulate both the stability and the vaporization thresholds of the PCNs. This discovery can have important implications for both nanoparticle delivery and potential therapeutic paradigms. First, modulating the stability of PCNs through these methods can potentially allow for the design of PCNs with controlled circulating half-lives based upon the specific application the PCNs for which they are intended. This is extremely desirable with regard to extravascular imaging and therapy where longer circulating times will be required for accumulation in the interstitial space. However, too much stability could also impair the efficiency of the particle vaporization. In other words, the nanoparticles may be able to be designed opti-
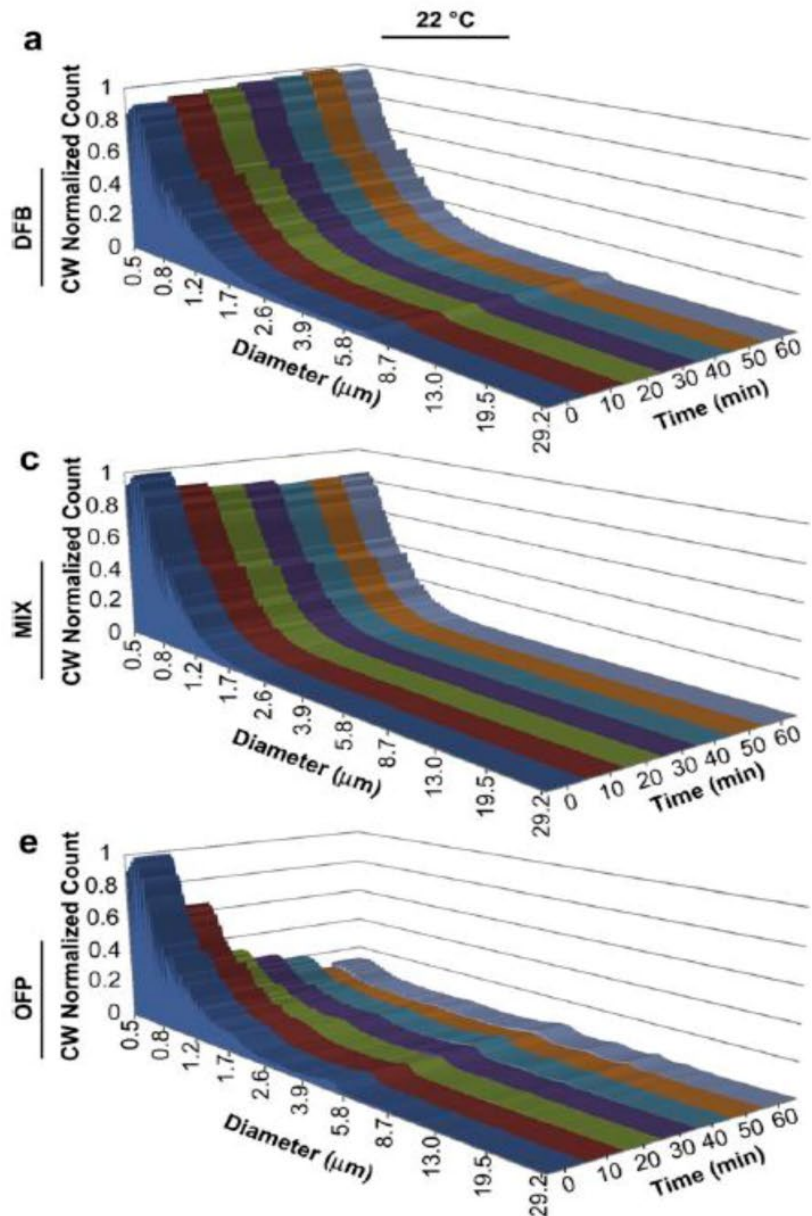

mally for the competing aspects of delivery, imaging, and therapeutic manipulation. Second, if the vaporization thresholds of these PCNs can be modulated, we may be able to apply only as much ultrasound as is required to image or elicit therapeutic effect, thereby avoiding potential bioeffects. Third, an additional advantage of being able to modulate these PCNs would be to allow for optimizing the emulsions for specific aspects of separation of different components of ultrasound mediated delivery (i.e. radiation force and acoustic streaming), vaporization (i.e. conversion from nanoparticle to microbubble for imaging), and finally cavitation (inertial or stable cavitation of resultant microbubble) for the purposes of therapeutic manipulations (i.e. vascular occlusion, sonothrombolysis, and intracellular ablation). Experiments are continuing in our laboratories, as well as others, to specifically address these possibilities.
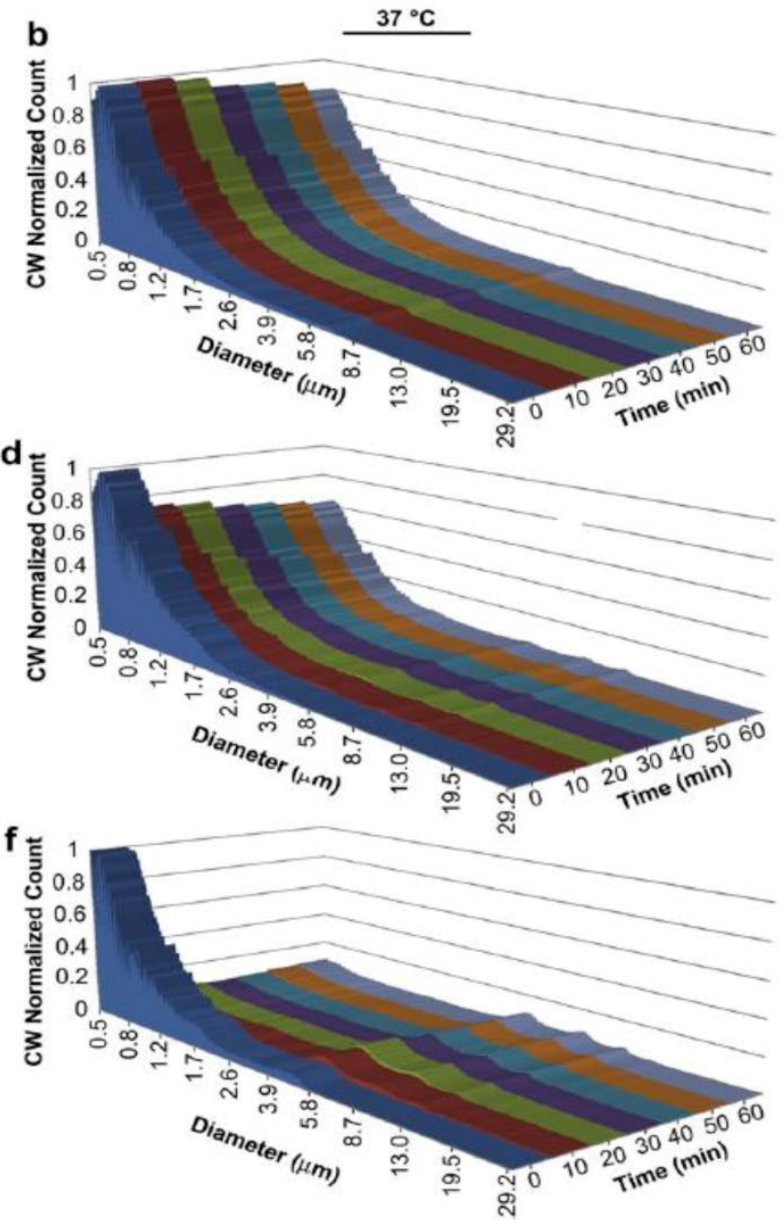

Figure 8. Perfluorocarbon droplet distributions in vitro over a I h period: Pure DFB at a) $22 \mathrm{C}$ and b) $37 \mathrm{C}$; DFB. OFP mixture at c) 22 $\mathrm{C}$ and d) $37 \mathrm{C}$; and pure octafluoropropane at e) $22 \mathrm{C}$ and f) $37 \mathrm{C}$. The distribution at each time point was scaled to the relative mean concentration (concentration-weighted) to simultaneously reflect changes in concentration over the time period. Reprinted from Biomaterials. Vol. 33. Sheeran PS, Luois S, Mullin LB et al. Design of ultrasonically-activatable nanoparticles using low boiling point perfluorocarbons. pgs. 3262-3268, Copyright 20II, with permission from Elsevier. 


\section{Targeted Delivery}

Since PCNs formed by microbubble condensation have lipid membranes stabilizing their surface, they are also amenable to decoration by targeting ligands on their membrane surface - the same strategy commonly applied to microbubble targeting schemes [52]. We demonstrated the ability to develop PCNs targeted to cellular receptors/biomarkers for the purposes of molecular imaging using a cyclic RGD peptide conjugated to a lipid-PEG component on the shell $[53,54]$. Utilizing human umbilical vein endothelial cells (HUVECs) known to upregulate $\alpha_{\mathrm{v}} \beta_{3}$ receptors on the membrane surface, PCNs were incubated for 15 minutes with cells grown on acoustically transparent Thermanox ${ }^{\circledR}$ plates [55]. Samples were then exposed to ultrasound energy at $8 \mathrm{MHz}(\mathrm{MI}=$ 1.1) delivered via a clinical ultrasound machine (Acuson Sequoia 512, 15L8 linear array transducer) to mediate vaporization, and imaged with the same machine at low acoustic energy $(7 \mathrm{MHz}, \mathrm{MI}=0.18)$ in both B-mode and contrast-specific CPS mode to measure the resulting increase in image contrast due to the PCNs. Results (see Figure 9) indicate a statistically significant increase $(p=0.02)$ in binding of targeted droplets over control, sham-targeted droplets registered as a six-fold improvement in mean ultrasound intensity after vaporization to microbubbles. Figure 10 shows a volumetric rendering of individual B-mode scans (greyscale) with contrast-specific signal overlays (greenscale). After the vaporization pulse, a region of high contrast is visible in the HUVEC cell layer due to adherent post-vaporization microbubbles on the surface of the cells, demonstrating that the vaporized bubbles remain attached to the HUVEC cellular membrane surface and do not float to the surface of the covering slide. This suggests that the newly formed microbubbles will likely retain their encapsulating shell to some degree, and that the targeting ligands will maintain their adherence during PCN-to-microbubble conversion - enabling ADV-based molecular imaging. Thus, ADV-based molecular imaging may have significance for both vascular and extravascular applications due to the fact that, once bound to a receptor target, sufficient clearance time to both the vascular and extravascular space can be afforded such that only the adherent particles will be seen after activation.

\section{Clinical/Translational Implications and Future Directions}

The use of PCNs may have widespread application and could significantly change the breadth of targeted microbubble agents to include imaging or delivery to extravascular targets as opposed to being limited to intravascular flow or targeting endothelial biomarkers. Below are descriptions of just a few potential new areas of research that may be pursued with these new PCNs based on highly volatile perfluorocarbons.

\section{Tumor Cell Ablation, a non-pharmacologic approach}

Extravascular delivery of volatile PCNs in the interstitial space can potentially be accompanied by vaporization and/or cavitation of resultant microbubbles to aid in the ablation of regions of pathology (i.e. tumor cells). Currently, there are reports of PCNs acting as nucleation sites for HIFU tumor ablation [35]. In addition, Kang and Yeh have reported the successful delivery of perfluoropentane PCNs to the intracellular compartment of macrophages (referred to as drug-loaded macrophages) followed by ADV and subsequent inertial cavitation. They propose the use of macrophages as transporters of the PCNs to the extravascular space [56]. Low boiling point PCNs may have the potential to activate these droplets more efficiently and enhance the effect even more with less inherent peripheral bioeffects than HIFU.

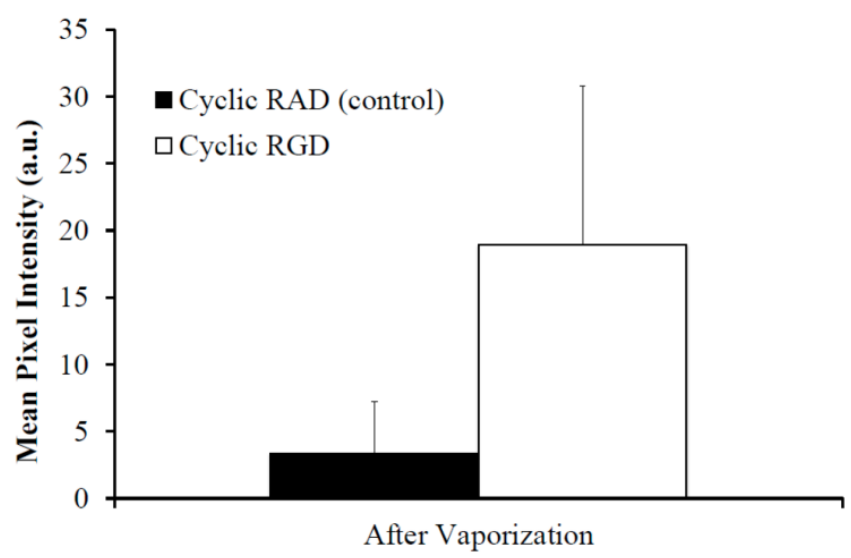

Figure 9. Mean scattering intensity as a function of PCN binding to HUVEC cells. Note the significant increase in scattering intensity with targeted $\mathrm{PCNs}$ vs. untargeted $\mathrm{PCNs}$, demonstrating that the cyclic RGD ligand targets $\alpha v \beta 3$ expression in the HUVEC cells, and that the PCNs are capable of providing quantitative information on molecular expression at the cellular level. (C) 2012 IEEE. Reprinted, with permission from P.S. Sheeran et al., "Ultrasound molecular imaging with customizable nanoscale phase-change contrast agents: an in-vitro feasibility study", 2012 IEEE International Ultrasonics Symposium (IUS) Proceedings, In Press, 2012. 


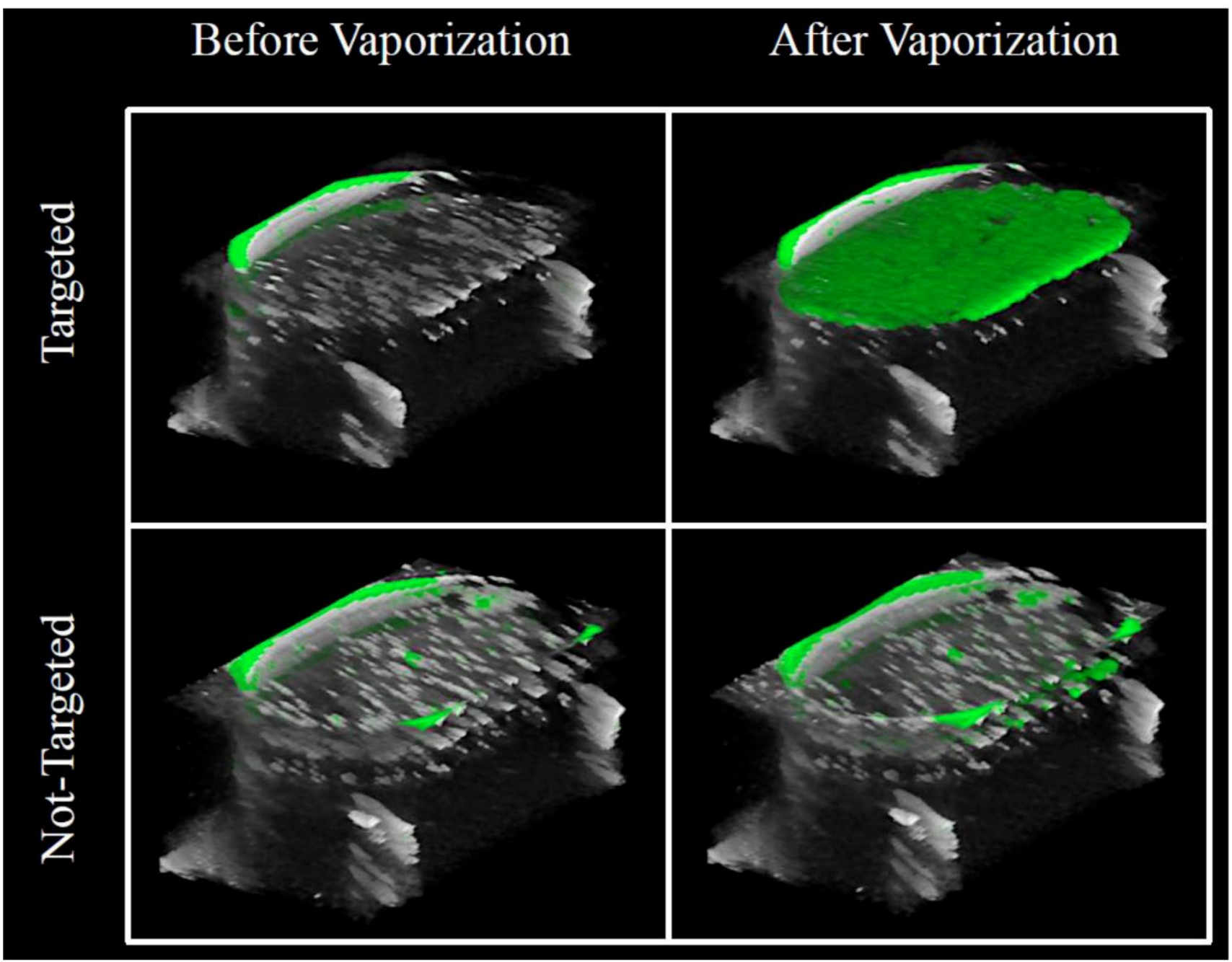

Figure 10. Overlays of contrast-specific CPS (green) and B-mode (grey) ultrasound scans of HUVEC cells incubated with targeted PCNs: Prior to activation, no contrast-specific echogenicity is detected, suggesting no microbubbles have formed. After exposure to a mechanical index of I.I at $8 \mathrm{MHz}$, targeted droplets vaporize to the highly-echogenic microbubble state and remain in the plane of the HUVEC cells. *note: rarefactional pressure (in $\mathrm{MPa}$ ) can be calculated from the mechanical index with $P_{\text {neg }}=M I \times \sqrt{8}$. (C) 2012 IEEE. Reprinted, with permission from P.S. Sheeran et al., "Ultrasound molecular imaging with customizable nanoscale phase-change contrast agents: an in-vitro feasibility study", 2012 IEEE International Ultrasonics Symposium (IUS) Proceedings, In Press, 2012.

Alternatively, nanoparticle delivery to the intracellular space followed by vaporization may offer unique opportunities for tissue and tumor cell ablation provided that the particles can be situated near regions where sub-cellular damage can induce an apoptotic or lethal event. Thus, a possible area of interest would be to deliver PCNs to cellular surfaces followed by intracellular uptake and targeting to subcellular organelles (i.e. via nuclear localization sequences for example). As an example, therapeutics can be conjugated to nuclear localization sequences such that upon intracellular delivery (endocytosis or membrane diffusion), a migration to the nucleus can be achievable for enhanced tumor destruction $[57,58]$.
Similar methods may be feasible for intracellular delivery of PCNs followed by imaging and/or ablation. This may provide the opportunity for a theranostic approach whereby microbubbles resulting from droplet vaporization may be utilized for imaging tumor cells selectively from the intracellular milieu as well as following tumor regression if cells can be ablated by either ;1) radiation force secondary to particle vaporization, or; 2) cavitation of the microbubble. It is also possible that cellular destruction could be achieved by the combination of the two. Thus, by modulating the ultrasound output, one may be able to generate a sufficient "shockwave" to compromise the target, thereby eliciting sufficient damage to induce 
apoptosis. In addition, since the ablation could potentially occur from the intracellular compartment of the tumor cell, this could lead to selective tumor cell ablation with a minimum of bioeffects to surrounding cells. It would be expected that the shockwave would dissipate quickly over space and primarily effect the targeted cell. This hypothesis is supported by data, which suggests bubble-induced cell membrane effects are greatest within one bubble diameter of the cell, and fall off rapidly at greater distances [59].

\section{Sonothrombolysis}

Despite the potential utility for extravascular application, PCNs may also be utilized for intravascular delivery for imaging and therapy of conditions such as vascular thrombi. Currently, microbubbles have been explored for this purpose [60-62], however, an increase in circulation time plus the benefit of activating at a specific site may make PCNs a viable alternative to microbubbles for the purposes of Sonothrombolysis. Because their size is in the 100s of nm range, they may offer advantages over microbubbles for permeating into the thrombus matrix, at which point they may be activated and even cavitated for lysing the clot. In addition, by virtue of their size and surface pegylation, PCNs may be less subject to elimination than microbubbles. As such, they may offer increased utility as a therapeutic agent for the purposes of acute clot dispersion. Finally, due to the use of lower ultrasound output for vaporization, they may potentially offer significant improvements over current sonothrombolytic therapies especially in terms of decreased adverse effects as the effect would be elicited primarily through the "shockwave" imparted from the vaporization of the droplet to the microbubble as opposed to the subsequent microbubble eliciting an effect through inertial cavitation. Current evidence indicates that this would be the case as the rationale for using vaporization with low-boiling perfluorocarbons is that the transition from droplet to microbubble requires less energy than microbubble cavitation.

\section{Gene and Drug Delivery}

The flexibility of the formulation method and the ability to enhance delivery to the interstitial space by the enhanced permeability and retention (EPR) effect opens opportunities for more efficient ultrasound-mediated gene therapy directed to specific tissues. Because droplets can be generated with cationic shells, the opportunity for electrostatic decoration of the nanoparticle shell with plasmids is indeed possible. Furthermore, the modification of the formulation with mixtures of perfluorocarbons (both volatile and less volatile) provides the means to modulate the ultrasound requirements for ADV. This also implies that one could adjust vaporization potential such that we may be able to delineate separate ultrasound requirements for imaging, radiation force and acoustic streaming, vaporization, and microbubble cavitation - which should aid in more controlled delivery, activation, and microbubble cavitation to promote gene and drug delivery.

\section{Characterization of Enhanced Permeabil- ity and Retention Effect}

The effects of leaky endothelial gap junctions and the resultant increased diffusion of small molecule drugs have been addressed previously. However, this effect, despite a good deal of precedence, has been difficult to quantify. PCNs, by virtue of their ability to potentially extravasate, may conceivably be activated in the region of the tumor tissue space followed by vaporization and quantitating ultrasound backscatter from resultant microbubble formation. The quantitation might then be used as an indication of the number of PCNs that have diffused into the tissue space. Thus, one may be able to quantify the level of extravasation as a function of the passive diffusion of nanoparticles of known size into the region of interest. This could be somewhat helpful with respect to determining the ability of passive diffusion of nanoparticle delivery agents in the region of angiogenesis [11, 13]. Consequently, a general determination of the 'treatability' potential of a tumor via ADV agents could lead to optimization of dosage based upon tumor burden and angiogenic vessel permeability.

\section{Lymphatic Imaging}

Previous studies have shown the ability to use microbubbles for sentinel node imaging as an indicator of cancer spread and disease progression [63, 64]. Some effort has focused upon the use of microbubbles to image lymphatics in the in the stomach and in peritumoral regions of cancer [65, 66]. Thus, lymphatic imaging could be amenable to PCN studies as well, and may be aided by the small particle size compared to microbubble agents.

\section{Theranostics}

As described above, the localization and vaporization of a PCN intracellularly offers the opportunity to create a therapeutic response and to be able to image the particular cell simultaneously. Thus, it is possible that one could monitor the progress of therapy using localized, intracellularly deposited PCNs and using ultrasound imaging while at the same time, 
initiate therapy and follow regression of the disease.

\section{Imaging of Digestive Organs}

An area of interest that may be explored is the imaging of the digestive organs. Although transabdominal ultrasound imaging of the alimentary tract is difficult secondary to luminal gas artifacts, endoscopic ultrasound overcomes this by imaging through water instilled in the lumen or by imaging through a water-filled balloon attached to the tip of the endoscope [67-70].

The esophagus is of particular interest, as it is easily accessible, can be filled with fluid and is the organ that houses a common cancer with an increasing incidence [71]. Adenocarcinoma of the esophagus is a complication of Barrett's esophagus, where the normal squamous epithelium is replaced by intestinal metaplasia in response to gastroesophageal reflux $[72,73]$. The progression from Barrett's to esophageal cancer occurs through stages of low and high grade dysplasia, which are not visible to the naked eye. Currently, blind four quadrant biopsies are performed at one to two $\mathrm{cm}$ intervals, which sample less than five percent of the Barrett's mucosa and are thus open to sampling error [74]. This is particularly worrisome as the smallest areas of high grade dysplasia can be no more than a few $\mathrm{mm}$ in diameter). Ligands have now been identified that enable areas of high grade dysplasia and cancer to be imaged with fluorescent antibodies $[75,76]$. Targeted attachment of PCNs via small ligands will enable the dysplastic and cancerous cells to be located, but additionally help to facilitate targeted destruction of such cells, without the pain and complications of non-selective therapy [77]. Furthermore, following targeted ablation, patients can be surveyed for evidence of remaining or recurrent dysplasia and retreated by a technique that will be highly selective and thus more effective and with few complications.

A second application of such techniques would be in the bile and pancreatic ducts that are commonly accessed and cannulated during endoscopy. The attachment of PCNs to targets such as Claudin-4 and mixed matrix-metaloperoxidase-7 that are expressed in dysplasia and cancer could enable neoplastic strictures of bile and pancreatic ducts to be imaged and ablated [78, 79]. Similarly, such techniques can be used during surgery to assist the surgeon excise all microscopic cancer, which is currently not possible, for a substantial improvement in long-term survival [80].

\section{Conclusion}

Phase-change nanoparticles may show promise as potential extravascular contrast agents if we are able to induce vaporization at acceptable ultrasound outputs so as to elicit the desired response without the undesirable bioeffects. As future work continues to mature, PCNs may show promise as vascular and enteral contrast agents as well. Work by others has demonstrated the utility and potential of perfluorocarbon liquid particles for imaging and therapeutic applications [81, 82]. The stabilization of highly volatile perfluorocarbons may help to bridge the applications into the extravascular space. Indeed, further work is still necessary in this area however, it has the potential to expand the realm of ultrasound contrast agents to develop approaches to interstitial tissue imaging and therapy that is currently only available to other modalities. Furthermore, potential advances in nanoparticle delivery to regions of the intracellular space, enteral space, and lymphatics may offer the opportunity for additional theranostic paradigms to be developed. Finally, although much future work is needed, maturation of any of the above areas opens the door for highly translational projects to develop into clinical realities.

\section{Acknowledgements}

The authors appreciate the support of the NIH through R21EB011704 (TOM), R01EB008733 (PAD), the Carolina Center for Cancer Nanotechnology Excellence (PAD), and a graduate fellowship from the National Science Foundation (PSS) in supporting their work on acoustically activated nanoparticles.

\section{Abbreviations}

SPECT: Single-photon emission computed tomography. PET: Positron emission tomography. MRI: Magnetic Resonance imaging. MCA: Microbubble-based contrast agents. UCA: Ultrasound contrast agents. PFC: Perfluorocarbon. DFB: Decafluorobutane. OFP: Octafluoropropane. DDFP: Dodecafluoropentane. PFH: Perfluorohexane.

\section{Competing Interests}

The authors have declared that no competing interest exists.

\section{References}

1. Gramiak R, Shah PM. Echocardiography of the aortic root. Invest Radiol. 1968; 3: $356-66$

2. Porter TR, Xie F. Transient myocardial contrast after initial exposure to diagnostic ultrasound pressures with minute doses of intravenously injected microbubbles. Demonstration and potential mechanisms. Circulation 1995; 92: 2391-5.

3. Wei K, Jayaweera AR, Firoozan S, et al. Quantification of myocardial blood flow with ultrasound-induced destruction of microbubbles administered as a constant venous infusion. Circulation. 1998; 97: 473-83. 
4. Chomas JE, Pollard RE, Sadlowski AR, et al. Contrast-enhanced US of microcirculation of superficially implanted tumors in rats. Radiology. 2003; 229: 439-46.

5. Miller DL, Thomas RM. Ultrasound contrast agents nucleate inertial cavitation in vitro. Ultrasound Med Biol. 1995; 21:1059-65.

6. Lawrie A, Brisken AF, Francis SE, et al. Microbubble-enhanced ultrasound for vascular gene delivery. Gene Ther. 2000; 7: 2023-7.

7. Tachibana K, Tachibana S. Albumin microbubble echo-contrast material as an enhancer for ultrasound accelerated thrombolysis. Circulation. 1995; 92: 1148-50.

8. Hernot S, Klibanov AL. Microbubbles in ultrasound-triggered drug and gene delivery. Adv Drug Deliv Rev. 2008; 60: 1153-66.

9. Gessner R, Dayton PA. Advances in molecular imaging with ultrasound. Mol Imaging. 2010; 9: 117-27.

10. Kircher HF, Willmann JK. Molecular Body Imaging: MR Imaging, CT, and US. Part I. Principles. Radiology. 2012; 263: 233-43.

11. Campbell RB. Tumor physiology and delivery of nanopharmaceuticals. Anticancer Agents Med Chem. 2006; 6: 503-12.

12. Hobbs SK, Monsky WL, Yuan F, et al. Regulation of transport pathways in tumor vessels: Role of tumor type and microenvironment. Proc Natl Acad Sci USA. 1998; 95: 4607-12.

13. Torchilin VP. Passive and active drug targeting: Drug delivery to tumors as an example. Handb Exp Pharmcol. 2010; 197: 3-53.

14. Quaia E. Contrast media in ultrasonography-basic principles and clinical applications. New York: Springer. 2005.

15. Streeter JE, Gessner R, Miles I, et al. Improving sensitivity in ultrasound molecular imaging by tailoring contrast agent size distribution: in vivo studies. Molecular imaging 2010; 9: 87-95.

16. Talu E, Hettiarachchi $K$, Zhao S, et al. Tailoring the size distribution of ultrasound contrast agents: possible method for improving sensitivity in molecular imaging. Mol Imaging. 2007; 6: 384-92.

17. Gorce JM, Arditi M, Schneider M. Influence of bubble size distribution on the echogenicity of ultrasound contrast agents: a study of SonoVue. Invest Radiol. 2000; 35: 661-71.

18. Martinez HP, Kono Y, Blair SL, et al. Hard shell gas-filled contrast enhancement particles for colour Doppler ultrasound imaging of tumors. Med Chem Comm. 2010; 1: 266-270.

19. Lee SJ, Schlesinger PH, Wickline SA, et al. Interaction of melittin peptides with perfluorocarbon nanoemulsion particles. J Phys Chem B. 2011; 115: 15271-9.

20. Behan M, O'Connell D, Mattrey R, et al. Perfluorooctylbromide as a contrast agent for CT and sonography: preliminary clinical results. Am J Roentgenol. 1993; 160: 399-405.

21. Lanza G, Winter $P$, Caruthers $S$, et al. Theranostics for tumor and plaque angiogenesis with perfluorocarbon nanoemulsions. Angiogenesis. 2010; 13: 189-202.

22. Lanza GM, Wickline SA. Targeted ultrasonic contrast agents for molecular imaging and therapy. Progr Cardiovasc Dis. 2001; 44: 13-31.

23. Mattrey RF. Perfluorooctylbromide: a new contrast agent for CT, sonography, and MR imaging. Am J Roentgenol. 1989; 152: 247-52.

24. Xing $\mathrm{Z}$, Wang $\mathrm{J}$, Ke $\mathrm{H}$, et al. The fabrication of novel nanobubble ultrasound contrast agent for potential tumor imaging. Nanotechnology. 2010; 21: 145607.

25. Dayton PA, Matsunaga TO. Ultrasound-Mediated Therapies Using Oil and Perfluorocarbon-filled Nanoparticles. In: Moos W.H and Barry S.E, eds. Drug Development Research. Hoboken, NJ: Wiley-Liss, Inc. 2006: 42-6

26. Hendrickson GR, Lyon LA. Microgel Translocation Through Pores under Confinement. Angew Chem. 2010; 49: 2193-97.

27. Kripfgans OD, Orifici CM, Carson PL, et al. Acoustic droplet vaporization for temporal and spatial control of tissue occlusion: A kidney study. IEEE Trans Ultrason Ferroelectr Freq Cont. 2005; 52: 1101-10.

28. Zhang M, Fabiili ML, Haworth KJ, et al. Initial investigation of acoustic droplet vaporization in canine kidney. Ultrasound Med Biol. 2010; 36: 1691-703.

29. Sheeran PS, Dayton PA. Phase-change contrast agents for imaging and therapy. Curr Pharm Des. 2012; 18: 2152-65.

30. Apfel R. Activatable Infusable dispersions containing drops of a superheated liquid for methods of therapy and diagnosis. US Patent No.5,840,276. Nov 24, 1998

31. Rapoport NY, Kennedy AM, Shea JE, et al. Controlled and targeted tumor chemotherapy by ultrasound-activated nanoemulsions/microbubbles. J Cont Rel. 2009; 138: 268-76.

32. Rapoport NY, Efros AL, Christensen DA et al. Microbubble generation in phase-shift nanoemulsions used as anticancer drug carriers. Bubble Sci Eng Technol. 2009; 1: 31-39.
33. Quay SC. Phase shift colloids as ultrasound contrast agents. US Patent No.5,558,853. Sept. 24, 1996.

34. Kawabata KI, Sugita N, Yoshikawa H, et al. Nanoparticles with multiple perfluorocarbons for controllable ultrasonically induced phase shifting. Jpn J Appl Phys. 2005; 44: 4548-52.

35. Zhang P, Porter T. An in vitro study of a phase-shift nanoemulsion: a potential nucleation agent for bubble-enhanced HIFU tumor ablation. Ultrasound Med Biol. 2010; 36 : 1856-66.

36. Sheeran PS, Wong VP, Luois S, et al. Decafluorobutane as a phase-change contrast agent for low-energy extravascular ultrasound imaging. Ultrasound Med Biol. 2011; 37: 1518 - 30.

37. Kripfgans OD, Fowlkes JB, Miller DL, et al. Acoustic droplet vaporization for therapeutic and diagnostic applications. Ultrasound Med Biol. 2000; 26: 1177-89.

38. Reznik N, Williams R, Matsuura N, et al. Perfluorocarbon nanodroplets for extravascular contrast. In The 17th Annual Symposium on Ultrasound Contrast Agents; Jan.19-20, 2012. Rotterdam: The Netherlands.

39. Martz TD, Bardin D, Sheeran PS, et al. Microfluidic Generation of Acoustically Active Nanodroplets. Small 2012; 8: 1876-9.

40. Giesecke T, Hynynen K. Ultrasound-mediated cavitation thresholds of liquid perfluorocarbon droplets in vitro. Ultrasound Med Biol. 2003; 29: 1359-65.

41. Kabalnov, A. Ostwald ripening and related phenomena. J Disp Sci Tech. 2001; 22: 1-12.

42. Sirsi SR, Chen CC, Feshitan JA, et al. Development of "Bulky" Lipid Microbubbles for Ultrasound Triggered Drug Delivery. Nashville, TN: AIChE Annual Meeting. 2009.

43. Sheeran PS, Luois S, Dayton PA, et al. Formulation and acoustic studies of a new phase-shift agent for diagnostic and therapeutic ultrasound. Langmuir 2011; 27: 10412-20.

44. Feshitan JA, Chen CC, Kwan JJ, et al. Microbubble size isolation by differential centrifugation. Journal of Colloid and Interface Science, 2009; 329: 316-24

45. Reznik N, Williams R, Burns PN. Investigation of Vaporized Submicron Perfluorocarbon Droplets as an Ultrasound Contrast Agent. Ultrasound Med Biol. 2011; 37: 1271-9.

46. Lundgren CE, Bergoe GW, Tyssebotn I. The theory and application of intravascular microbubbles as an ultra-effective means of transporting oxygen and other gases. Undersea Hyperb Med. 2004; 31: 105-6.

47. Lundgren CE, Bergoe GW, Tyssebotn IM. Intravascular fluorocarbon-stabilized microbubbles protect against fatal anemia in rats. Artif Cells Blood Subst Immobil Biotechnol. 2006; 34: 473-86.

48. Lundgren C, Bergoe G, Olszowka A, et al. Tissue nitrogen elimination in oxygen-breathing pigs is enhanced by fluorocarbon-derived intravascular micro-bubbles. Undersea Hyperb Med. 2005; 32: 215-26.

49. Reznik N, Seo M, Williams R, et al. Optical studies of vaporization and stability of fluorescently labeled perfluorocarbon droplets. Phys Med Biol. 2012; 57: 7205-17.

50. Prosperetti, A. Bubble phenomena in sound fields. Part one. Ultrasonics. 1984; 22: 69-78

51. Sheeran PS, Luois SH, Mullin LB, et al. Design of ultrasonically-activatable nanoparticles using low boiling point perfluroocarbons. Biomaterials. 2012; 33: 3262-9.

52. Klibanov AL, Microbubble contrast agents: targeted ultrasound imaging and ultrasound-assisted drug-delivery applications. Invest Radiol. 2006; 41: 354-62.

53. Sheeran PS, Streeter JE, Mullin LB, et al. Ultrasound Molecular Imaging with Customizable Nanoscale Phase-Change Contrast Agents: An In-Vitro Feasibility Study. IEEE International Ultrasonics Symposium (IUS) Proceedings. October 7-10, 2012; Dresden Germany.

54. Sheeran PS, Streeter JE, Mullin LB et al. Toward Ultrasound Molecular Imaging with Phase-Change Contrast Agents: An In-Vitro Proof-of-Principle. Ultrasound Med Biol.; in press.

55. Dayton PA, Pearson D, Clark J, et al. Ultrasonic analysis of peptide- and antibody-targeted microbubble contrast agents for molecular imaging of avß3-expressing cells. Mol Imaging 2004; 3: v125-34.

56. Kang S-T, Yeh C-K. Intracellular Acoustic Droplet Vaporization in a Single Peritoneal Macrophage for Drug Delivery Applications. Langmuir 2011; 37: 13183-8.

57. Wang HY, Chen JX, Sun YX, et al. Construction of cell penetrating peptide vectors with $\mathrm{N}$-terminal stearylated nuclear localization signal for targeted delivery of DNA into the cell nuclei. J Control Release. 2011; 155: 26-33.

58. Wang HY, Li C, Yi WJ, et al. Targeted delivery in breast cancer cells via iodine: nuclear localization sequence conjugate. Bioconj Chem. 2011; 22: 1567-75. 
59. Zhou Y, Yang K, Gao S, et al. Controlled permeation of cell membrane by single bubble acoustic cavitation. J Contr Rel. 2012; 157: 103-11.

60. Xie F, Slikkerveer J, Gao S et al. Coronary and microvascular thrombolysis with guided diagnostic ultrasound and microbubbles in acute ST segment elevation myocardial infarction. J Am Soc Echocardiogr. 2011; 24: $1400-8$.

61. Hennings LJ, Flores R, Roberson PK, et al. Persistent Penumbra in a Rabbit Stroke Model: Persistence and Histologic Characteristics. Stroke Res Treat. 2011; epub.

62. Alexandrov AV, Mikulik R, Ribo M, et al. A Pilot Randomized Clinical Safety Study of Sonothrombolysis Augmentation With Ultrasound-Activated Perflutren-Lipid Microspheres for Acute Ischemic Stroke. Stroke. 2008; 39: 1464-9.

63. Wisner, ER, Ferrara, KW, Short RE et al. Sentinel Node Detection using Contrast-Enhanced Power Doppler Ultrasound Lymphography. Invest Radiol. 2003; 38(6): 358-365.

64. Goldberg, BB, Merton, DA, Liu, J-B et al. Contrast-Enhanced Ultrasound Imaging of Sentinel Lymph Nodes After Peritumoral Administration of Sonazoid in a Melanoma Tumor Animal Model. J Ultrasound Med. 2011; 30: $441-53$.

65. Goldberg BB, Merton DA, Liu JB, et al. Contrast-enhanced sonographic imaging of lymphatic channels and sentinel lymph nodes. Ultrasound Med Biol. 2005; 24: 953-65.

66. Kawai Y, Aijima K, Nagai T, et al. Real-time imaging of the lymphatic channels and sentinel lymph nodes of the stomach using contrast-enhanced ultrasonography with Sonazoid in a porcine model. Cancer Sci. 2011; 102: 2073-81.

67. Klapman J, Chang KJ, Wiersema M, et al. Endoscopic ultrasound-guided fine-needle aspiration biopsy in esophageal cancer. Endoscopy Apr 2005; 37: 381-5.

68. Lambert R, Caletti G, Cho E, et al. International Workshop on the clinical impact of endoscopic ultrasound in gastroenterology. Endoscopy. 2000; 32: 549-84.

69. Kahaleh M, Shami VM, Conaway MR, et al. Endoscopic ultrasound drainage of pancreatic pseudocyst: a prospective comparison with conventional endoscopic drainage. Endoscopy. 2006; 38: 355-9.

70. Caletti G, Fusaroli P. The rediscovery of endoscopic ultrasound (EUS) in gastric cancer staging. Endoscopy 2012; 44: 553-5.

71. Shaheen NJ. Advances in Barrett's esophagus and esophageal adenocarcinoma. Gastroenterology 2005; 128: 1554-66.

72. Sharma P, McQuaid K, Dent J, et al. A critical review of the diagnosis and management of Barrett's esophagus: the AGA Chicago Workshop. Gastroenterology 2004; 127: 310-30.

73. Spechler SJ, Sharma P, Souza RF, et al. American Gastroenterological Association medical position statement on the management of Barrett's esophagus. Gastroenterology. 2011; 140: 1084-91.

74. Chatelain D, Flejou JF. High-grade dysplasia and superficial adenocarcinoma in Barrett's esophagus: histological mapping and expression of p53, p21 and Bcl-2 oncoproteins. Virchows Arch. 2003; 442: 18-24.

75. Li M, Anastassiades CP, Joshi B, et al. Affinity peptide for targeted detection of dysplasia in Barrett's esophagus. Gastroenterology 2010; 139: $1472-80$.

76. Goetz M, Wang TD. Molecular imaging in gastrointestinal endoscopy. Gastroenterology. 2010; 138: 828-33.

77. Bulsiewicz WJ, Shaheen NJ. The role of radiofrequency ablation in the management of Barrett's esophagus. Gastrointest Endosc Clin N Am. 2011; 21: 95-109.

78. Crawford HC, Scoggins CR, Washington MK, et al. Matrix metalloproteinase-7 is expressed by pancreatic cancer precursors and regulates acinar-to-ductal metaplasia in exocrine pancreas. J Clin Invest. 2002; 109: 1437-44.

79. Nichols LS, Ashfaq R, Iacobuzio-Donahue CA. Claudin 4 protein expression in primary and metastatic pancreatic cancer: support for use as a therapeutic target. American journal of clinical pathology. 2004; 121 : 226-30.

80. Raut CP, Tseng JF, Sun CC, et al. Impact of resection status on pattern of failure and survival after pancreaticoduodenectomy for pancreatic adenocarcinoma. Ann Surg. 2007; 246: 52-60.

81. Rapoport NY, Nam KH, Gao Z et al. Application of Ultrasound for Targeted Nanotherapy of Malignant Tumors. Acoust Phys. 2009; 55: 594-601.

82. Fabiilli ML, Haworth KJ, Sebastian IE et al. Delivery of chlorambucil using an acoustically-triggered perfluoropentane emulsion. Ultrasound Med Biol. 2010; 36: 1364-75. 